\title{
Abdominal Aortic Aneurysm
}

National Cancer Institute

\section{Source}

National Cancer Institute. Abdominal Aortic Aneurysm. NCI Thesaurus. Code C27000.

Enlargement and ballooning of the vessel that supplies arterial blood to the abdomen, pelvis and legs. 